\title{
Natural vs. Vaccine Mediated COVID-19 Immunity Scientific Justification to Mandate Vaccination of Recovered Healthcare Workers
}

To protect the vulnerable patients, many healthcare facilities (including DHR Health System) have mandated COVID-19 vaccination of its healthcare workers, vendors, contractors, students, residents, and fellows. This policy has been widely debated and it has been suggested that healthcare facilities should adopt a different policy for workers who have recovered from natural SARS-CoV-2 infection and a waiver should be granted to them as far as COVID-19 vaccination mandates are concerned. It has been argued that individuals who have been previously infected with SARS-CoV-2 virus have acquired immunity which should provide protection against re-infection and therefore they are at a lower risk for the patient.

In the United States, over 40 million people have been infected with SARS-CoV-2 and over 378 million doses of COVID-19 vaccines have already been administered (1). Some people with COVID19 have very mild symptoms and they may see natural infection as preferable to receiving the COVID-19 vaccine. Additionally, there are some people who are concerned that getting a COVID-19 vaccine could make them sicker if they do ultimately get COVID-19. It is incontrovertible that getting infected with SARS-CoV-2 may offer some natural protection. Current evidence suggests that reinfection with the SARS-CoV-2 is uncommon in the 90 days after initial infection. However, it is unclear as to how long this protection lasts, and the risk of severe illness and death from COVID-19 far outweighs any benefits that are afforded by natural immunity (2). It was therefore recommended that even people who have been previously infected with SARS-CoV-2 must consider getting vaccinated.

In an independent study, sera obtained from recovered and naïve donors collected prior to, and following immunizations with existing mRNA vaccines, was tested to determine its neutralizing capacity against the wild-type and the SARS-CoV2 variant (3). Pre-vaccination sera from recovered donors neutralized the wild type but only intermittently neutralized the variant. On the contrary, a single dose of immunization boosted neutralizing titers against the receptor binding domain of both the variant and the wild type by up to 1000 -fold. This study underscores the importance of vaccinating both uninfected and previously infected persons to elicit cross-variant neutralizing antibodies.

The fact that vaccinated individuals are at reduced risk of infection as compared to patients who have recovered from SARS-CoV-2 infection was also confirmed by another case-control study (4). COVID-19 infections in Kentucky among people who were previously infected with SAR-CoV-2 shows that unvaccinated individuals are more than twice as likely to be reinfected with COVID-19 than those who were fully vaccinated after initially contracting the virus. This data further indicates that COVID-19 vaccines offer better protection than natural immunity alone and that vaccines, even after prior infection, help prevent reinfections.

The rapid spread of the Delta variant and the real potential for continued emergence of more transmissible and infectious SARS-CoV-2 mutations because of hesitancy in getting vaccinated poses a serious national challenge which demands implementation of an urgent plan of action. Consequently, on Thursday, September 09, 2021, the U.S., President issued a bold and aggressive plan entitled: PATH OUT OF THE PANDEMIC: PRESIDENT BIDEN'S COVID-19 $A C T I O N$ PLAN (5). This Plan has six distinct initiatives which, when successfully implemented would markedly reduce the risk associated with the spread of SARS-CoV-2 variants. Under the 
initiative entitled "Vaccinating the Unvaccinated", the following mandates were communicated:

- Requiring all employers with 100+ employees to ensure their workers are vaccinated or tested weekly

- Requiring vaccinations for all Federal workers and for millions of contractors that do business with the Federal government

- Requiring COVID-19 vaccinations for over 17 million health care workers at Medicare and Medicaid participating hospitals and other healthcare settings

- Calling on large entertainment venues to require proof of vaccination or testing for entry

- Requiring employers to provide paid time off to get vaccinated

While vaccinating recovered and naïve healthcare workers is essential, this mandate must be implemented ethically ensuring that healthcare workers fully understand both the empirical and moral rationale for the mandatory vaccination program. Simply imposing a mandate without laying the ethical groundwork is likely to result in measurable challenges. A prudently developed process must include educational and promotional campaigns to enhance healthcare worker's knowledge and understanding of the complex and rapidly evolving science related to COVID-19. This may include organizing Townhall meetings with relevant experts in a forum that allows the healthcare workers to ask questions anonymously. Furthermore, this mandate must be anchored on the compelling patient safety concerns and an opt-out criteria based on legitimate and verifiable religious beliefs or medical condition should be made available to the healthcare workers. To ensure its widespread acceptance, mandatory vaccination policies should be transparent and impartial ensuring that it is equally applicable to all members of the healthcare fraternity. Lastly, healthcare facilities should make every attempt to provide vaccinations that are free of charge and easily accessible.

In conclusion we recommend that based on available scientific evidence, existing U.S. Centers for Disease Control \& Prevention policies, and in the best interest of the patients, health care facilities are justified in requiring all its employees, vendors, contractors, and learners to be vaccinated even if they have recovered from previous infection with SARS-CoV-2.

\section{References}

1. Johns Hopkins Coronavirus Resource Center. COVID-19 Dashboard. https://coronavirus.jhu.edu/map.html (Accessed September 12, 2021; 22:19 hrs).

2. Centers for Disease Control \& Prevention. Answering Patients' Questions About COVID-19 Vaccine and Vaccination. https://www.cdc.gov/vaccines/covid-19/hcp/answeringquestions.html

3. Stamatatos L, Czartoski J, Wan YH, Homad LJ, Rubin V, Glantz H, Neradilek M, Seydoux E, Jennewein MF, MacCamy AJ, Feng J, Mize G, De Rosa SC, Finzi A, Lemos MP, Cohen KW, Moodie Z, McElrath MJ, McGuire AT. mRNA vaccination boosts cross-variant neutralizing antibodies elicited by SARSCoV-2 infection. Science. 2021 Mar 25: eabg9175. doi: 10.1126/science. abg9175. Epub ahead of print. PMID: 33766944; PMCID: PMC8139425.

4. Cavanaugh AM, Spicer KB, Thoroughman D, Glick C, Winter $\mathrm{K}$. Reduced risk of reinfection with SARS-CoV-2 after COVID19 vaccination - Kentucky, May - June 2021. MMWR Morb Mortal Wkly Rep 2021; 70: 1081-1083. https://www.cdc.gov/mmwr/volumes/70/wr/mm7032e1.htm?s $\mathrm{cid}=\mathrm{mm} 7032 \mathrm{e} 1 \mathrm{w}$

5. Path Out of the Pandemic: President Biden's Covid-19 Action Plan. Sept. 09, 2021. https://www.whitehouse.gov/covidplan/

\section{Sohail Rao, MD, MA, DPhil}

Executive Vice President, DHR Health, 5501 S. McColl Road, Edinburg, Texas

President \& Chief Executive Officer, DHR Health Institute for Research \& Development, 5323 S. McColl Road, Edinburg, Texas

Corresponding author email: s.rao@dhr-rgv.com

Disclosures: None

ORCID: Sohail Rao: https://orcid.org/0000-0001-5027$\underline{9992}$

Manish Singh, MD, FACS

Chief Executive Officer, DHR Health, 5501 S. McColl Road, Edinburg, Texas

Disclosures: None

ORCID: Manish Singh: https://orcid.org/0000-00034146-3282 